\title{
Delayed post-surgical sepsis from Teflon felt: The diagnostic value of CT scanning, and a reminder for theatre staff
}

\section{Donald Emby, MB BCh, FFRad (D) SA Andrew Lancaster, $M B$ ChB}

AngloGold Ashanti Health, Western Deep Levels Hospital, Carletonville

Corresponding author: D Emby (demby@anglogoldashanti.com)

\section{Introduction}

We report on 2 patients with surgical site infections following the inadvertent use of Teflon felt for haemostasis in elective and emergency surgery. CT scanning was superior to plain radiography in demonstrating the foreign bodies to enable planning of further surgical treatment.

\section{Case 1}

A 30-year-old woman presented to Casualty in February 2008 complaining of pain in her left ear and a discharging wound. She had previously had a left tympano-mastoidectomy for chronic suppurative otitis media in December 2007. On clinical examination, marked swelling of the soft tissues of the left temporal region and a chronically discharging sinus over her left mastoid were noted. She was otherwise well. A staphylococcus aureus was cultured from the wound discharge.

No abnormality was visible on plain film radiographs of the skull (Fig. 1a). A CT scan of the brain, however, demonstrated a large, folded, radio-dense foreign body within the soft tissues of the scalp in the left tempero-parietal region (Fig. 1b). At surgical exploration under general anaesthetic, two felt-like foreign bodies were removed with difficulty from ingrowing tissue. These were subsequently identified as Teflon felt.

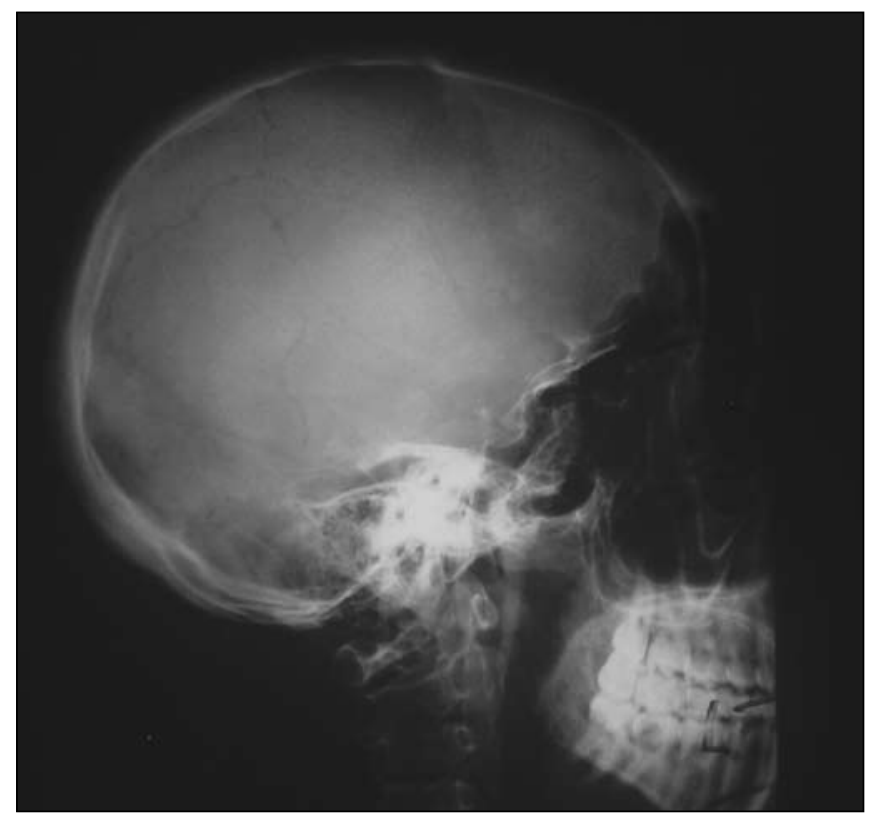

Fig. 1a. Lateral skull radiograph. Teflon felt is not visible

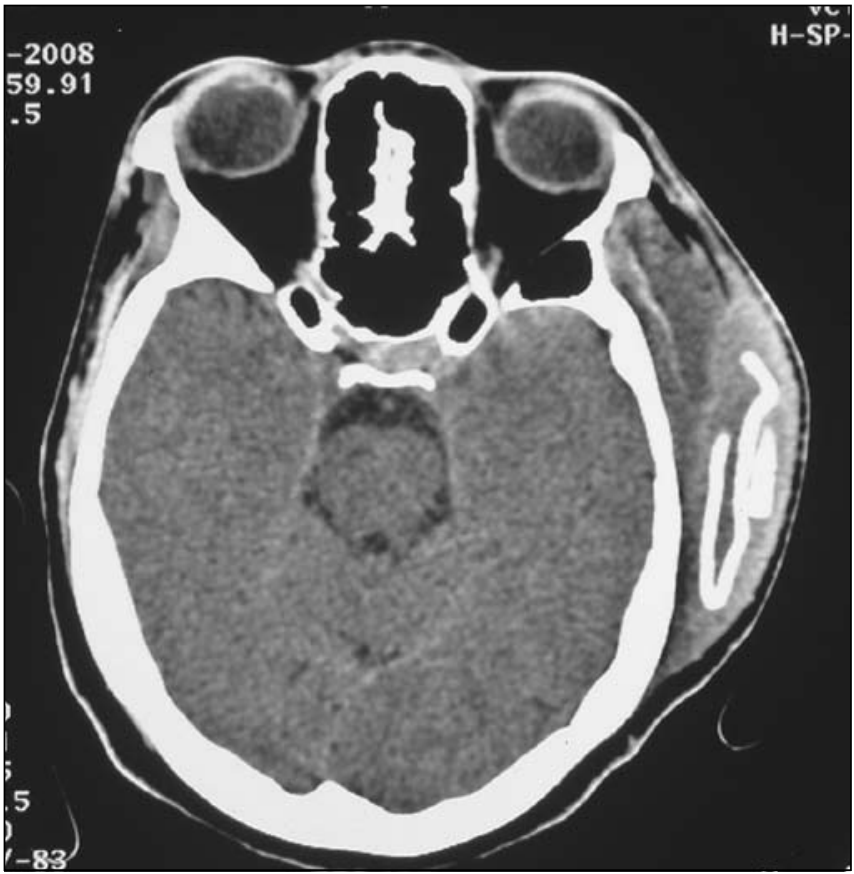

Fig. 1b. CT image showing Teflon felt in soft tissues of scalp.

The wound was irrigated and closed.

The patient received cloxacillin intravenously post-operatively for 2 days, then orally for 5 days. Complete healing was subsequently documented.

\section{Case 2}

A 45-year-old man was admitted to Casualty in December 2007 with a gunshot injury.

On examination, wounds to his right femoral triangle and the right side of his scrotum were noted. He was in Type 3 haemorrhagic shock from a haemorrhaging right femoral artery. The patient was urgently transferred to theatre where the right femoral artery was repaired, a right thigh fasciotomy performed, and the right-sided scrotal wound debrided. He required transfusion of 8 units of packed cells. He was discharged approximately 3 weeks later in mid-January 2008 .

The patient was re-admitted in March 2008 for drainage of a right thigh abscess. He subsequently developed chronic wound sepsis. Radiographs of his thigh showed no visible abnormality (Fig. 2a). Wound swabs grew Proteus mirabilis, methicillin-resistant Staphylococcus aureus and methicillin-resistant S. epidermedis. The wound sepsis settled with 


\section{CASE REPORT}

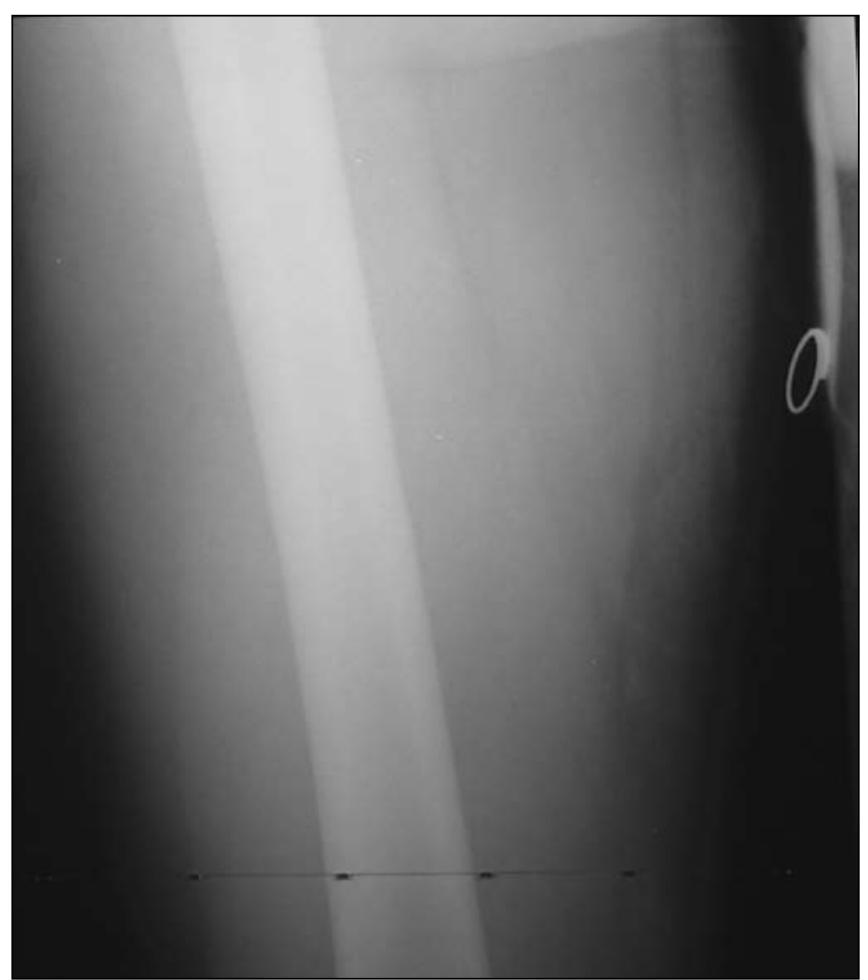

Fig. 2a. Right thigh with circular metallic skin-marker; Teflon felt not visible.

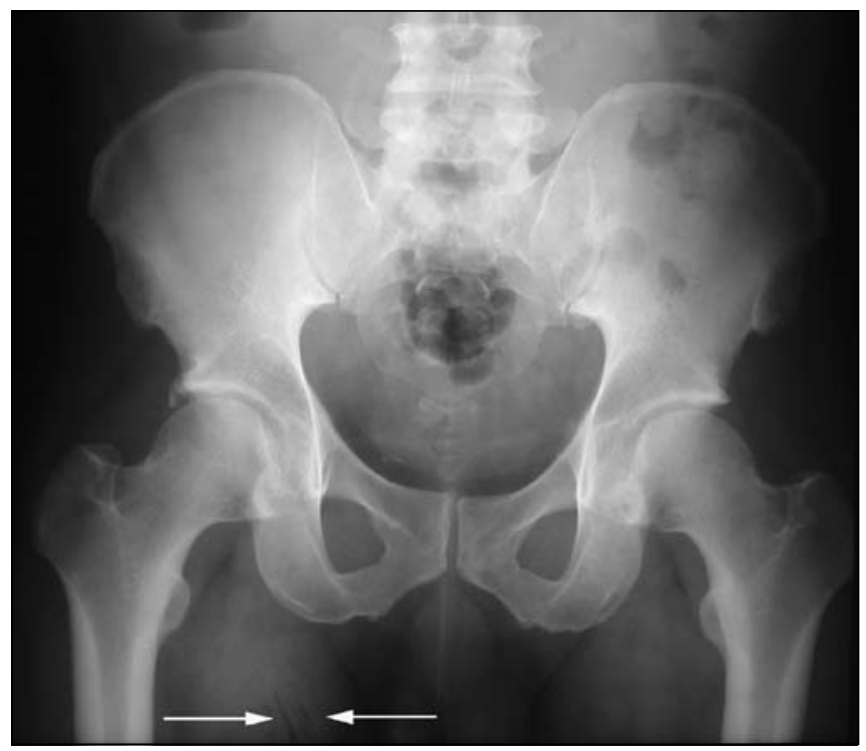

Fig. 2b. Teflon felt not visible in pelvic X-ray; note air lucencies in right medial thigh (arrows).

dressings. He was re-admitted in January 2010 with recurrence of the wound sepsis, with skin ulceration and a discharging sinus.

A radiograph of the pelvis including the upper thighs was obtained. This showed faint linear lucencies within the soft tissues of the medial thigh on the right (Fig. 2b). No radio-dense foreign body was visible. A CT scan of the right inguinal region and thigh was then performed and this showed a folded, radio-dense foreign body, extending over a long segment of the upper thigh (Fig. 2c). At surgical exploration under general anaesthetic, a $6 \times 4 \mathrm{~cm}$ piece of Teflon felt intimately associated with the old femoral artery repair was removed and the wound closed. The patient subsequently made a full recovery and returned to work.

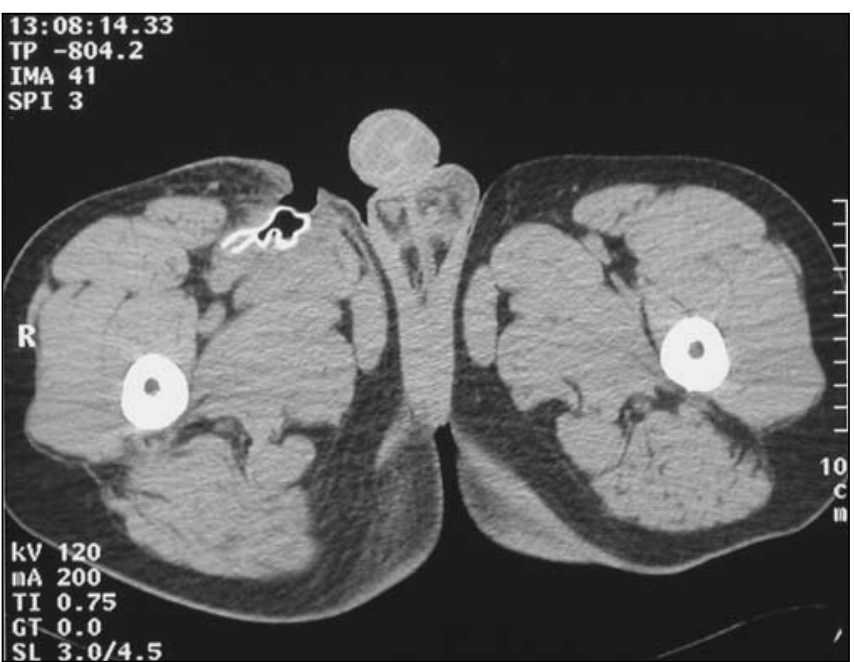

Fig. 2c. CT scan image through upper thighs shows Teflon felt in base of ulcer. Ulcer crater contains air; this accounts for the fine linear lucencies visible within the soft tissues of the right medial thigh on the pelvic radiograph.

\section{Discussion}

In both patients, non-absorbable PTFE (Teflon) felt had been inadvertently used for haemostasis in place of an absorbable haemostatic material, at the initial operation. Oxidised regenerated cellulose (Surgicel) fibrillar is the preferred absorbable material that is routinely used at this institution for fast haemostasis of bleeding tissue with irregular surface. Absorbable haemostatic gelatine sponge (Spongostan) (both from Johnson and Johnson Wound Management) is regarded as an acceptable alternative.

The absorbable haemostatic materials and (non-absorbable) Teflon felt are macroscopically similar in appearance and can be easily confused if theatre staff are inexperienced or are not aware of the important differences in their biological properties. In both cases, the inadvertent use of Teflon felt resulted in a foreign body response that became infected.

Textile implants are not biocompatible. These implants elicit a foreign body response that will either break down a non-stable polymer through enzymatic attack or hydrolysis, or will encapsulate a stable polymer with native tissue to isolate it. The foreign body response is what allows textile implants to become incorporated into tissue. ${ }^{1}$ Incorporation is facilitated if an implant is in the form of a mesh. Biocompatibility in terms of such implants really denotes that the implants are non-toxic. ${ }^{1}$ If the operative field is potentially infected (as in Case 1), or potentially contaminated (as in Case 2), the implantation of non-absorbable Teflon felt into the soft tissues results in a foreign body reaction that is prone to secondary infection.

Non-absorbable Teflon felt is intended for use in cardiac surgery, most commonly as a patch where there is a tissue defect but also as a buttress for sutures, using small pledgets for vessel repair. It can be used as a material for replacement of segments of ventricular myocardium after resection. ${ }^{2}$ Its use in the repair of left ventricular rupture has been well described. ${ }^{3}$

Of particular interest is that the Teflon felt was not visible on plainfilm radiographs but was prominently radio-dense on CT scan. This once again highlights the very much greater sensitivity of CT scanning compared with standard radiographs in the detection of low-density objects within tissues. Recognised examples of low-density objects that 


\title{
CASE REPORT
}

are not visible on standard radiographs but are clearly shown on CT scan include faintly calcified ureteric calculi and low-density foreign bodies such as certain types of glass ${ }^{4}$ and also fish bones. ${ }^{4}$

The two cases described clearly show that CT scanning is the modality of choice for demonstrating Teflon felt when this has been used as an implant. Following these incidents, a case can be made to lobby the manufacturers of Teflon felt to investigate colouring the felt in such a way that it is readily macroscopically distinguishable from other implantable materials.

1. Textiles in Medicine. http://surgicalmesh.com/sldindex_files/slide0001.htm (accessed 18 March 2010) 2. Bard PTFE Felt. http://www.bardpv.com/_vascular/product.php?p=30 (accessed 17 March 2010

3. de la Fuente A, Augudo O, Sanchez R, Fernandez JL, Moriones I. Repair of left ventricular rupture after mitral valve replacement: use of Teflon patch and glue. Ann Thoral Surg 1999;67:1082-1083. 4. Emby DJ. Retained glass fragments in body tissues. S Afr Med J 2009;99(12):858-859.

\section{Rupture of an ovarian artery aneurysm following normal vaginal delivery}

\author{
Francois van Schouwenburg, $M B C h B$ \\ Hassan Lameen, MB ChB, MD, FCRad (Diag) SA \\ Department of Radiology, Grey's Hospital, Pietermaritzburg
}

Corresponding author: H Lameen (h.alameen1@yahoo.com)

\section{Introduction}

Ruptured ovarian artery aneurysms are extremely rare, occurring during the peripartum or early postpartum periods. Only 11 cases have been reported in the English literature; the following represents the 12th.

\section{Case report}

A 30-year-old woman presented at the casualty department 12 days after having given birth to a healthy baby via normal vaginal delivery. She complained of increasing pain and a mass in her right flank. There were no complaints of nausea, vomiting, dysuria or abnormal bowel habits. On examination, the patient was acutely distressed, in severe pain and

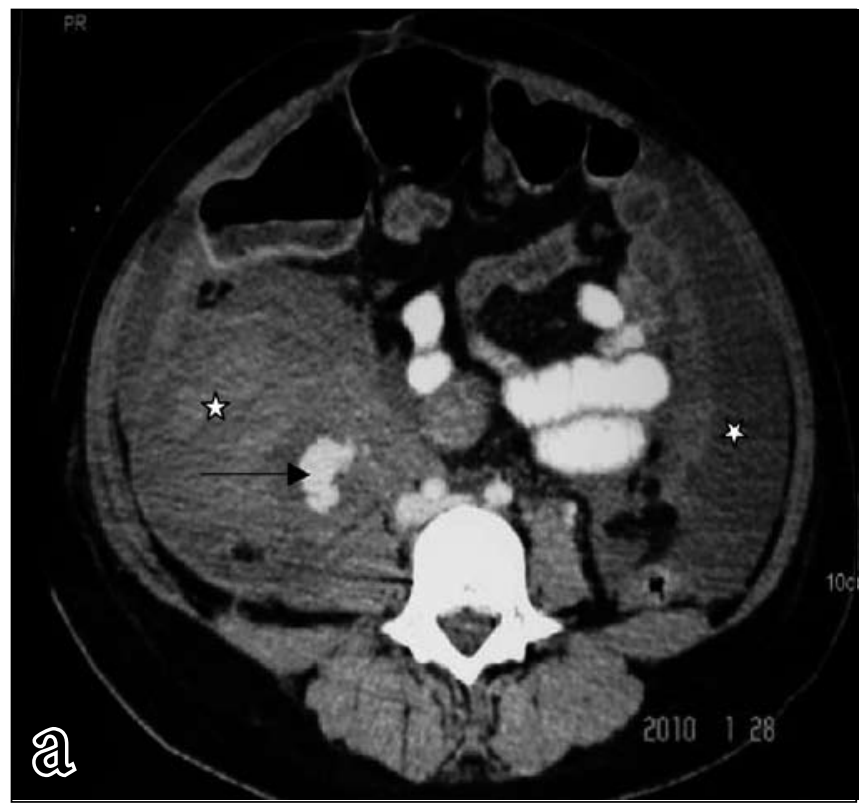

anaemic with an $\mathrm{Hb}$ of $5 \mathrm{~g} / \mathrm{dl}$. Her blood pressure was 151/71 $\mathrm{mmHg}$ and her pulse 125/minute. Physical examination revealed a severely distended abdomen with features of peritonitis.

Abdominal ultrasound revealed a large abdominal fluid collection; a CT scan was therefore requested. A contrast-enhanced CT scan demonstrated a large homogenous retroperitoneal fluid collection in the right flank, with a $25 \mathrm{~mm}$ rounded, intensely enhancing focus located close to the inferior border of the collection (Fig. 1). The collection displaced the bowel to the left and inferiorly, and the liver superiorly.

The differential diagnosis included a ruptured ovarian artery aneurysm. As soon as the patient was stable, she was taken for ovarian

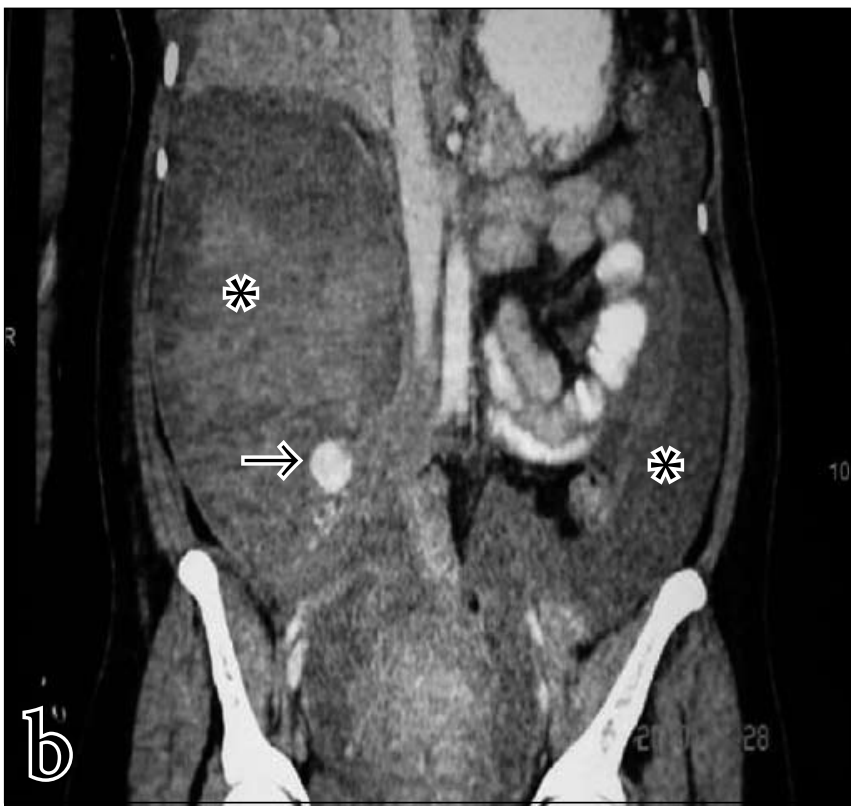

Figs $1 a$ and b. Axial and coronal contrast-enhanced CT image of the abdomen demonstrating large right retroperitoneal abdominal and pelvic haematoma (stars) with right-sided focal area of contrast extravasation at the expected location of the right ovarian artery (arrow). Ascites also present. 